\title{
X-RAY VARIABILITY COHERENCE: HOW TO COMPUTE IT, WHAT IT MEANS, AND HOW IT CONSTRAINS MODELS OF GX 339-4 AND CYGNUS X-1
}

\author{
Brian A. Vaughan ${ }^{1}$ and Michael A. NowaK ${ }^{2}$ \\ Received 1996 September 6; accepted 1996 September 30
}

\begin{abstract}
We describe how the coherence function-a Fourier frequency-dependent measure of the linear correlation between time series measured simultaneously in two energy channels-can be used in conjunction with energy spectra, power spectra, and time delays between energy channels to constrain models of the spectrum and variability of X-ray binaries. Here we present a procedure for estimating the coherence function in the presence of counting noise. We apply this method to the black hole candidates Cyg X-1 and GX 339-4 and find that the near-perfect coherence between low- and high-energy X-ray photons rules out a wide range of models that postulate spatially extended fluctuating emission, thermal flares, and overlapping shot noise.
\end{abstract}

Subject headings: black hole physics — methods: statistical — X-rays: stars

\section{INTRODUCTION}

Rapid $(T<1000 \mathrm{~s})$ aperiodic variability is common to all types of X-ray binaries. It arises in the immediate vicinity of the compact object and provides a probe of changes in physical parameters, such as accretion rate, optical depth, and temperature. Power spectra and cross spectra have been applied widely to study variability in X-ray binaries as a function of frequency and energy, as well as to measure time delays between intensity variations at different energies (cf. van der Klis 1989). Here we describe the coherence function-a measure of the degree of linear correlation between two time series as a function of Fourier frequency-and how this provides strong constraints on models of X-ray binaries, especially recent theoretical models that attempt to correlate energy spectra with aperiodic variability behavior (see Nowak 1994; Miyamoto et al. 1994; Miller 1995; Nowak \& Vaughan 1996). We expect the coherence function to be widely applicable to Rossi X-Ray Timing Explorer (RXTE) observations.

No current model mimics all facets of a system as complex as an X-ray binary. However, spectral and dynamical models should qualitatively reproduce-or, at the very least, not fundamentally disagree with—all of their most basic average properties: the energy spectrum, power spectrum (PSD), and time delays and coherence between different energies. (For ways in which a spectral model can disagree with timing data, see Miller 1995 and Nowak \& Vaughan 1996.) Most theories model the energy spectra, fewer model the PSD, fewer still consider phase lags, and, to date, none consider the coherence function. Here we describe several examples that lead to unity coherence, as well as several that generically lead to a loss of coherence.

We apply the coherence function to the black hole candidate Cyg X-1 in its low (hard) state and to GX 339-4 in its very high state. The former shows unity coherence over a wide range of Fourier frequencies and energy bands, whereas the latter shows a sharp drop in coherence between low- and high-energy bands. We present these data to show the appli-

${ }^{1}$ California Institute of Technology, MC 220-47, Pasadena, CA 91125; brian@srl.caltech.edu.

2 Joint Institute for Laboratory Astrophysics, University of Colorado, Campus Box 440, Boulder, CO 80309-0440; mnowak@rocinante.colorado.edu. cation of the statistical methods to real data and to show that there are intrinsic differences among distinct physical states and systems.

\section{COMPUTING THE COHERENCE FUNCTION}

Let $x_{1}(k)$ and $x_{2}(k)$ be the X-ray flux observed simultaneously in two energy channels at times $t_{k}$. The Fourier transforms of $x_{1}(k)$ and $x_{2}(k)$ at frequency $\nu_{j}$ are $X_{1}(j)$ and $X_{2}(j)$, respectively. We will usually use lowercase variables to indicate time series and uppercase variables to indicate Fourier transforms. From the Fourier transforms, one can construct the power spectra $\left[P_{1}(j)=\left|X_{1}(j)\right|^{2}\right.$ and $P_{2}(j)=$ $\left.\left|X_{2}(j)\right|^{2}\right]$ and the (complex-valued) cross spectrum $[C(j)=$ $X_{1}^{*}(j) X_{2}(j)$ ], whose argument is the phase difference between intensity fluctuations in the two channels at frequency $\nu_{j}$, which can be converted to a time delay $\delta t(j)=$ $\arg [C(j)] /\left(2 \pi \nu_{j}\right)$.

Measured X-ray light curves always contain noise. For most $\mathrm{X}$-ray observations, the dominant noise source is counting, or Poisson, noise. We denote signal by $s$ and noise by $n$, and we write $x_{1}(k)=s_{1}(k)+n_{1}(k), X_{1}(j)=S_{1}(j)+N_{1}(j)$, and likewise for channel 2 . Hereafter, we usually drop the explicit frequency dependence to simplify notation. For power spectra, Poisson noise adds to the signal, and $P_{1}=\left|S_{1}\right|^{2}+\left|N_{1}\right|^{2}$. The signal power, $\left|S_{1}\right|^{2}$, can be estimated by subtracting the Poisson noise, $\left|N_{1}\right|^{2}$, from the measured power.

We now consider the idealized case of two simultaneous random processes, 1 and 2, and let $s_{1}(t)$ and $s_{2}(t)$ be noiseless signals drawn from these processes. It is usually possible to find a linear transform $-h(\tau)$, called the transfer functionthat relates $s_{1}(t)$ and $s_{2}(t)$ via

$$
s_{2}(t)=\int_{-\infty}^{\infty} h(t-\tau) s_{1}(\tau) d \tau
$$

or, equivalently, $S_{2}(f)=H(f) S_{1}(f)$, where $S$ and $H$ are the Fourier transforms of $s$ and $h$. If $H(f)$ is the same for all realizations of the two processes, the processes are said to be coherent at frequency $f$. In that case, $|\langle C(f)\rangle|^{2}=$ $|H(f)|^{2}\left\langle\left|S_{1}(f)\right|^{2}\right\rangle^{2}=\left\langle\left|S_{1}(f)\right|^{2}\right\rangle\left\langle\left|S_{2}(f)\right|^{2}\right\rangle$, where angle brack- 
ets denote an average over an infinite set of signals from the same processes. The coherence function is then defined as

$$
\gamma_{I}^{2}(f) \equiv \frac{|\langle C(f)\rangle|^{2}}{\left\langle\left|S_{1}(f)\right|^{2}\right\rangle\left\langle\left|S_{2}(f)\right|^{2}\right\rangle},
$$

where we affix a subscript $I$ when computing the intrinsic coherence between noiseless signals. It is clear that when $S_{1}(f)$ and $S_{2}(f)$ are related by a linear transform, $\gamma_{I}^{2}(f)=1$. Coherence is a measure of the fraction of the rms amplitude of one process at a given frequency that can be predicted from the other by a linear transform (Bendat \& Piersol 1986). It is important to note that, unlike powers and time delays, coherence can only be computed for an ensemble of independent measurements. For noiseless measurements from a finite number, $m$, of independent samples, the statistical uncertainty of the coherence function is $\delta \gamma_{I}=$ $(2 / m)^{1 / 2}\left(1-\gamma_{I}^{2}\right) /\left|\gamma_{I}\right|$ (Bendat \& Piersol 1986).

The coherence function of real data is given by analogy to equation (2), except that angle brackets are used to denote an average over a finite number, $m$, of independent measurements. The coherence function of noisy data will always be less than unity. The intrinsic coherence can be estimated by correcting each term in the discrete analogy to equation (2) for counting noise. For the powers in the denominator, one sets $\left|S_{1}\right|^{2}=P_{1}-\left|N_{1}\right|^{2}$, and similarly for $\left|S_{2}\right|$, where $P_{1}$ and $P_{2}$ are the measured, noisy power spectra of the signals.

$$
\text { Writing out }|\langle C\rangle|^{2} \text { in terms of signal and noise yields }
$$$$
|\langle C\rangle|^{2}=\left|\left\langle S_{1}^{*} S_{2}\right\rangle+\left\langle S_{1}^{*} N_{2}\right\rangle+\left\langle N_{1}^{*} S_{2}\right\rangle+\left\langle N_{1}^{*} N_{2}\right\rangle\right|^{2} .
$$

The first term is the average cross spectrum of the signals. Poisson noise in one channel is uncorrelated with Poisson noise in the other channel and with signal in either channel. Thus $|\langle C\rangle|^{2}$ is the squared magnitude of the sum of $S_{1}^{*} S_{2}$ and a complex random variable, $s$, whose real and imaginary parts each have zero mean and variance

$\operatorname{var} \varsigma=\frac{1}{2}\left(\left|S_{1}\right|^{2}\left|N_{2}\right|^{2}+\left|N_{1}\right|^{2}\left|S_{2}\right|^{2}+\left|N_{1}\right|^{2}\left|N_{2}\right|^{2}\right) / m$.

We define $s^{2} \equiv\left|\left\langle S_{1}^{*} S_{2}\right\rangle\right|^{2}, a^{2} \equiv|\langle C\rangle|^{2}$, and $n^{2} \equiv\left(\left|S_{1}\right|^{2}\left|N_{2}\right|^{2}+\right.$ $\left.\left|N_{1}\right|^{2}\left|S_{2}\right|^{2}+\left|N_{1}\right|^{2}\left|N_{2}\right|^{2}\right) / m$. The probability distribution of the measured cross spectrum, $|\langle C\rangle|^{2}$, given an intrinsic cross spectrum and Poisson noise, is equivalent to the distribution of total power in an individual frequency bin of a noisy power spectrum; it is given by

$$
p\left(a^{2} \mid s^{2}, n^{2}\right)=n^{-2} \exp \left[\frac{-\left(a^{2}+s^{2}\right)}{n^{2}}\right] I_{0}\left(\frac{2 a s}{n^{2}}\right)
$$

(Groth 1975; Goodman 1985), where $I_{0}$ is the zero-order Bessel function of the first kind. This can be inverted to yield the probability distribution of $s^{2}$ given a measured cross spectrum and Poisson noise,

$p\left(s^{2} \mid a^{2}, n^{2}\right)=\frac{(n s)^{-2}}{\sqrt{\pi}} \exp \left[\frac{-\left(a^{2}+2 s^{2}\right)}{2 n^{2}}\right] \frac{I_{0}\left(2 a s / n^{2}\right)}{I_{0}\left(a^{2} / 2 n^{2}\right)}$

(Chakrabarty 1996). For $a \gg n$, equation (5) can be approximated by the Gaussian

$$
p\left(a^{2} \mid s^{2}, n^{2}\right) \approx \frac{1}{2 n a \sqrt{\pi}} \exp \left[\frac{-(a-s)^{2}}{n^{2}}\right],
$$

in which case $\left\langle a^{2}\right\rangle=\left\langle s^{2}\right\rangle+\left\langle n^{2}\right\rangle$ and $\delta a^{2}=\sqrt{2} n^{2}$. The intrinsic coherence can be usefully estimated when the following condi- tions are met: $s \gtrsim n,\left|S_{1}\right|^{2} /\left|N_{1}\right|^{2} \gtrsim 1 / \sqrt{m}$, and $\left|S_{2}\right|^{2} /\left|N_{2}\right|^{2} \gtrsim$ $1 / \sqrt{m}$.

The following is a recipe for estimating the value and uncertainty of the intrinsic coherence from measurements of $\langle C\rangle, P_{1}$, and $P_{2}$. Average powers and cross spectra should be constructed from unnormalized measurements. In practice, it will often be necessary to correct the Poisson noise level for instrumental dead time (see van der Klis 1989 for a detailed discussion). Dead time can introduce correlations between energy channels that enhance or diminish coherence. These are instrument dependent and beyond the scope of this Letter. The terms high power and high measured coherence used below denote powers that satisfy $|S|^{2}$ greater than a few times $|N|^{2} / \sqrt{m}$ in each channel and measured coherence that satisfies $\gamma^{2}$ greater than a few times $n^{2} /\left(P_{1} P_{2}\right)$, respectively. In many cases, these conditions will be satisfied at some frequencies but not at others.

High powers, high measured coherence.-

$$
\begin{aligned}
\gamma_{I}^{2}= & \frac{|\langle C\rangle|^{2}-n^{2}}{\left|S_{1}\right|^{2}\left|S_{2}\right|^{2}} \\
& \times\left\{1 \pm m^{-1 / 2}\left[\frac{2 n^{4} m}{\left(|\langle C\rangle|^{2}-n^{2}\right)^{2}}\right.\right. \\
& \left.\left.+\frac{\left|N_{1}\right|^{4}}{\left|S_{1}\right|^{4}}+\frac{\left|N_{2}\right|^{4}}{\left|S_{2}\right|^{4}}+\frac{m \delta \gamma_{I}^{2}}{\gamma_{I}^{4}}\right]^{1 / 2}\right\} .
\end{aligned}
$$

This is the Gaussian limit, and in essence, it corresponds to optimally filtering the measured coherence. The first three terms in the uncertainty come from uncertainties in Poisson noise, and the last term is from the statistical uncertainty in the intrinsic coherence.

High powers, low measured coherence.-Use equation (6) to determine confidence limits, $\left|\left\langle S_{1}^{*} S_{2}\right\rangle\right|_{\text {min }}^{2}$ and $\left|\left\langle S_{1}^{*} S_{2}\right\rangle\right|_{\text {max }}^{2}$, on $\left|\left\langle S_{1}^{*} S_{2}\right\rangle\right|^{2}$. Confidence limits on $\gamma_{I}^{2}$ are then

$$
\begin{aligned}
\gamma_{I, \min }^{2 \max }= & \frac{\left|\left\langle S_{1}^{*} S_{2}\right\rangle\right|^{2 \max }}{\left|S_{1}\right|^{2}\left|S_{2}\right|^{2}} \\
& \times\left[1 \pm\left(\frac{\left|N_{1}\right|^{4}}{m\left|S_{1}\right|^{4}}+\frac{\left|N_{2}\right|_{I}^{4}}{m\left|S_{2}\right|^{4}}+\frac{\delta \gamma^{2}}{\gamma_{I}^{4}}\right)^{1 / 2}\right] .
\end{aligned}
$$

Low powers.-This difficult case arises in weak sources and at high frequency. In practice, the $1 \sigma$ errors in this case are likely to extend nearly from 0 to 1 . The authors do not know a closed form for $p\left(\gamma_{I}^{2}\right)$. We recommend using equation (6) and the Gaussian probability distributions of $\left|S_{1}\right|^{2}$ and $\left|S_{2}\right|^{2}$ to empirically map out $p\left(\gamma_{I}^{2}\right)$ and determine confidence limits on the intrinsic coherence.

\section{EXAMPLES OF INCOHERENT SOURCES}

Thermal flares. - Let us imagine that the observed variability is completely the result of local temperature fluctuations with some time dependence, $T(t)$, which can be arbitrarily complicated so long as it is statistically stationary. Let the intrinsic time series, $s_{1}(t)$ and $s_{2}(t)$, be photon count rates in narrow frequency bands $\left(\nu_{1}, \nu_{1}+\delta \nu_{1}\right)$ and $\left(\nu_{2}, \nu_{2}+\delta \nu_{2}\right)$. The observed count rate in frequency band 1 is approximately proportional to $\nu_{1}^{2} \delta \nu_{1}\left(\exp \left[h \nu_{1} / k T(t)\right]-1\right)^{-1}$, and likewise for energy band 2. If both $h \nu_{1}$ and $h \nu_{2} \ll k T(t)$, then we are on the Rayleigh-Jeans portion of the spectrum, in which case $s_{1}(t) \propto s_{2}(t) \propto T(t)$. Thus there is a linear transfer function (a 
constant) between the two channels, and therefore, the coherence function will be unity. On the other hand, if $k T(t) \ll h \nu_{1}$ and $h \nu_{2}$, then we are on the Wien tail of the spectrum, in which case we have $s_{2}(t) \propto s_{1}(t)^{\left(\nu_{2} / \nu_{1}\right)} \propto \exp \left[-h \nu_{2} / k T(t)\right]$. Thus there is a nonlinear transfer function. In general, such a transfer function will take power from a frequency $f$ and distribute it among harmonics of $f$ (cf. Bendat \& Piersol 1986) and therefore lead to a loss of coherence.

Multiple flaring regions. - A number of models for X-ray variability in Galactic black hole candidates associate different timescales with different physical regions of an accretion disk (see Nowak 1994; Miyamoto et al. 1994). If more than one region contributes to the signal in both energy bands, then it is possible for the coherence function to be less than unity, even if individual regions produce perfectly coherent variability.

Consider two flaring regions: one produces a time series $q(t)=q_{1}(t)+q_{2}(t)$, and the other produces a time series $r(t)=r_{1}(t)+r_{2}(t)$ - each in energy bands 1 and 2, respectively. Furthermore, assume that there is a constant linear transfer function that relates $q_{1}(t)$ to $q_{2}(t)$, as well as another constant, linear transfer function that relates $r_{1}(t)$ to $r_{2}(t)$, with $q_{1}(t)$ and $r_{1}(t)$ otherwise being completely uncorrelated. Denoting the intrinsic time series observed in band 1 as $s_{1}(t)=$ $q_{1}(t)+r_{1}(t)$ and the intrinsic time series observed in band 2 as $s_{2}(t)=q_{2}(t)+r_{2}(t)$, the intrinsic coherence function between the two bands is then

$$
\gamma_{I}^{2}=\frac{Q_{1}^{2} Q_{2}^{2}+R_{1}^{2} R_{2}^{2}+2\left|Q_{1}\left\|Q_{2}\right\| R_{1} \| R_{2}\right| \cos \left(\delta \theta_{r}-\delta \theta_{q}\right)}{Q_{1}^{2} Q_{2}^{2}+R_{1}^{2} R_{2}^{2}+Q_{1}^{2} R_{2}^{2}+Q_{2}^{2} R_{1}^{2}},
$$

where $\delta \theta_{q}$ and $\delta \theta_{r}$ are the mean Fourier phase differences between $Q_{1}(f)$ and $Q_{2}(f)$ and between $R_{1}(f)$ and $R_{2}(f)$, respectively. (The quantities on the right-hand side eq. [10] refer to their mean values.) One can easily show that for this case, $\gamma_{I}^{2} \leq 1$ and is only equal to unity if both $\delta \theta_{q}=\delta \theta_{r}$ and $\left|Q_{1}\right| /\left|Q_{2}\right|=\left|R_{1}\right| /\left|R_{2}\right|$. That is to say, the coherence function is unity if and only if the same linear transfer function that takes $q_{1}(t)$ to $q_{2}(t)$ also takes $r_{1}(t)$ to $r_{2}(t)$.

\section{EXAMPLES OF COHERENT SOURCES}

Here we present an idealized model that produces unity coherence while producing phase lags qualitatively similar to those seen in Cyg X-1 (i.e., phase lags approximately independent of Fourier frequency; Miyamoto et al. 1992). Imagine that we have a disk with a stochastic source of (linear) surface density perturbations at the center that propagate outward at some constant speed $c_{s}$. If we take the source to be given by $(4 \pi r)^{-1} \delta(r) \sigma_{s}(t)$, then the resultant surface density perturbations- $\sigma_{p}(r, t)$, at radius $r$ and time $t$ (using the two-dimensional wave propagation Green's function; cf. Morse \& Feschbach 1953)—are given by

$$
\sigma_{p}(r, t)=\int d t^{\prime} \sigma_{s}\left(t^{\prime}\right) \frac{\theta\left[\left(t-t^{\prime}\right)-r / c_{s}\right]}{\sqrt{\left(t-t^{\prime}\right)^{2}-r^{2} / c_{s}^{2}}},
$$

where $\theta$ is the step function. Let the fluctuating signal in band $1, s_{1}(t)$, be equal to the surface density perturbation times a weighting function, $g_{1}(r)$, integrated over the entire disk. The radially integrated Green's function is just a linear transfer function; thus the Fourier transform of $s_{1}(t)$ becomes

$$
S_{1}(f)=\Sigma_{s}(f) \int i 2 \pi^{2} r g_{1}(r) H_{0}^{(1)}\left(\frac{2 \pi|f| r}{c_{s}}\right) d r,
$$

which we define as $\Sigma_{s}(f) T_{1}(f)$, and where $H_{0}^{(1)}(x)$ is the Hankel function of $x$ as well as the Fourier transform of the two-dimensional Green's function. Similarly, we have $S_{2}(f) \equiv$ $\Sigma_{s}(f) T_{2}(f)$. As both $S_{1}$ and $S_{2}$ are linearly related to the source $\Sigma_{s}$, there is a linear transfer function equal to $T_{2} / T_{1}$ that takes $S_{1}$ to $S_{2}$, and therefore, there is unity coherence. We could write such a transfer function only because the driving source was separable in time and space (in this case being spatially localized).

Equation (12) is analytically tractable if we set $g_{1}(r)=$ $\exp \left(-\alpha_{1} r / r_{0}\right)$ and $g_{2}(r)=\exp \left(-\alpha_{2} r / r_{0}\right)$, as one might have for weighting functions representing flux from a Wien tail. As will be discussed elsewhere (Vaughan et al. 1997), the resulting time lags are qualitatively similar to those seen in Cyg X-1. We require $r_{0} / c_{s} \sim 1$ to obtain quantitative agreement with the observations, which implies an extremely slow propagation speed, $c_{s} \sim 10^{-4} c$. This mechanism is qualitatively similar to that proposed by Manmoto et al. (1996), where thermal waves are launched from large disk radii toward the center and then reflect from the disk inner edge as acoustic waves. However, the mechanism of Manmoto et al. (1996) cannot preserve coherence if, as in the example of $\$ 3$, there are multiple, spatially distributed source functions (in the $X$-ray-emitting regions) for the waves. Furthermore, the acoustic waves shock upon reflection, which is an inherently nonlinear process that also will lead to a loss of coherence if emission from the reflected shock is an appreciable fraction of the total observed emission.

Adding additional mechanisms in the form of successive transfer functions will lead to unity coherence so long as each component is itself linear. In the above example, we could have invoked Comptonization as a subsequent transfer mechanism, which would have introduced additional time delays. So long as the Compton cloud is static, coherence will be preserved (cf. Nowak \& Vaughan 1996).

\section{OBSERVATIONAL EXAMPLES}

Of the above physical situations where coherence is preserved and those where it is not, which cases are observed in nature? Surprisingly, the answer seems to be that unity coherence is the norm, despite the variety of ways of weakening coherence. Here we present two black hole candidates: GX 339-4 in its very high state, and Cyg X-1 in its low state. Both have been modeled with disks plus Compton coronae (cf. Miyamoto et al. 1991; Dove, Wilms, \& Begelman 1996 and references therein), although there are differences between them: the very high state has a substantial soft $(\sim 1 \mathrm{keV})$ component, the low state has none; the hard tail of the very high state is softer than the hard tail of the low state $(\sim 2.5$ photon index compared to 1.7 ); the very high state has lower variability ( $\mathrm{rms} \sim 10 \%-20 \%$ ) than the low state (rms $\sim 40 \%$ ). Yet, as is shown in Figure 1, both systems apparently have unity intrinsic coherence, at least between low energies and at low frequency.

Figure 1 presents coherence plots derived from Ginga data. The methods described above have been used to filter the noise from the data. Poisson noise and uncertainties in the 


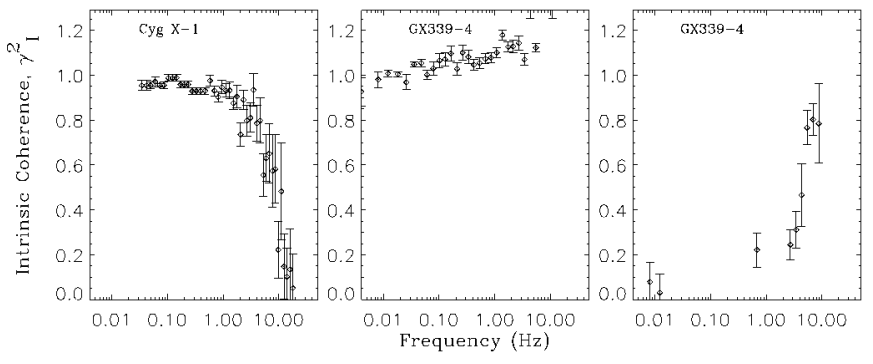

FIG. 1.-Coherence plots derived from Ginga data of Cyg X-1 in its low state (left, $1-2.3 \mathrm{keV}$ vs. 9.2-11.5 keV) and GX 339-4 in its very high state (middle, $2.3-4.6 \mathrm{keV}$ vs. $4.6-9.2 \mathrm{keV}$; right, $2.3-4.6 \mathrm{keV}$ vs. $13.7-37.1 \mathrm{keV}$ ). The high-frequency rollover in Cyg X-1 is likely the result of a misestimation of the Poisson noise level. Coherence exceeding unity in GX 339-4 may be the result of dead-time effects. The rapid coherence rise on the right is at the quasiperiodic oscillation frequency (cf. Miyamoto et al. 1991).

Ginga dead time make the coherence estimates unreliable above $\sim 10 \mathrm{~Hz}$; however, at frequencies below $10 \mathrm{~Hz}$, the intrinsic coherence is essentially unity. This result holds true for Cyg X-1 over a wide range of energy channels. On the other hand, the GX 339-4 variability (the PSD is shown as Fig. $4 b$ of Miyamoto et al. 1991) shows unity coherence between low energies but shows a dramatic drop in coherence between the 2.3-4.6 and 13.9-37.1 keV channels.

Considering the mechanisms discussed above, this loss of coherence is not surprising. Perhaps the 13.9-37.1 keV band corresponds to a Wien tale and, therefore, to a nonlinear transfer function. It is more difficult to explain the observed unity coherence. We expect that most shot models (cf. Lochner et al. 1991) will produce less than unity coherence. For example, the kinematic model of Nowak (1994) for the very high state of GX 339-4 successfully reproduced the observed PSDs and phase lags. However, as the viscous and thermal fluctuations were distributed over a large range of radii and overlapped in time, the mechanism led to less than unity coherence (not shown in that paper) between all energy bands. In order to preserve coherence, we always require linear responses, and we usually require the following: localized sources and/or localized responses, a (temporally) uniform source throughout the disk, or a uniform response throughout the disk. Most of these features are absent from current models.

The data described above will be presented in greater detail in Vaughan et al. (1997). Here we have presented them to demonstrate that there are observational and, by extension, physical differences between the systems, despite the fact that they are both commonly fitted with coronae models. The coherence function therefore offers us another tool to help distinguish among theoretical models.

\section{CONCLUSIONS}

We have described a statistic, the coherence function, that is derivable from the cross spectrum used to compute phase or time lags between two time series. This statistic has traditionally been ignored; however, it contains additional information about the system, and the methods described above can minimize the noise effects. Whenever one calculates the phase lag, one can and should also calculate the coherence function.

In general, coherence will be lost whenever there is a nonlinear transfer function between two channels or whenever there are multiple, uncorrelated (linear or nonlinear) transfer functions between two channels. However, our experience has been that coherence is preserved more often than not. This is a challenge for theoretical models, as there are many more mechanisms for destroying coherence than there are for preserving coherence.

We presented data from two black hole candidates: GX 339-4 in its very high state and Cyg X-1 in its low state. At low frequency and low energy both preserve coherence, although GX 339-4 loses coherence between high- and low-energy bands. Theoretical models have addressed observations of phase and time lags (e.g., Nowak 1994; Manmoto et al. 1996), as measured with EXOSAT, Ginga, and other X-ray timing instruments. No model currently accounts for the coherence properties detected in GX 339-4 and Cyg X-1. Forthcoming $R X T E$ observations, however, coupled with the tools presented above, can help provide new insights into the physical mechanisms at work in these systems, as well as new challenges for theorists to meet.

The authors would like to acknowledge useful conversations with Peter Michelson, Michiel van der Klis, Mitch Begelman, James Dove, and Jörn Wilms, as well as generous support from NASA grants NAG 5-3239 (B. A. V.) and NAG 5-3225 (M. A. N.) and the Netherlands Organization for Scientific Research (NWO) under grant PGS 78-277.

\section{REFERENCES}

Bendat, J., \& Piersol, A. 1986, Random Data: Analysis and Measurment Procedures (New York: Wiley)

Chakrabarty, D. 1996, Ph.D. thesis, California Inst. Tech.

Dove, J., Wilms, J., \& Begelman, M. C. 1996, ApJ, submitted

Goodman, J. W. 1985, Statistical Optics (New York: Wiley)

Groth, E. J. 1975, ApJS, 29, 285

Lochner, J. C., et al. 1991, ApJ, 376, 295

Manmoto, T., Takeuchi, M., Mineshige, S., Matsumoto, R., \& Negoro, H. 1996, ApJ, 464, L135

Miller, M. C. 1995, ApJ, 441, 770

Miyamoto, S., Kimura, K., Kitamoto, S., Dotani, T., \& Ebisawa, K. 1991, ApJ, 383, 784
Miyamoto, S., Kitamoto, S., Iga, S., \& Hayashida, K. 1994, ApJ, 435, 398 Miyamoto, S., Kitamoto, S., Iga, S., Negoro, H., \& Terada, K. 1992, ApJ, 391, L21

Morse, P. M., \& Feschbach, H. 1953, Methods of Theoretical Physics (New York: McGraw-Hill)

Nowak, M. A. 1994, ApJ, 422, 688

Nowak, M. A., \& Vaughan, B. A. 1996, MNRAS, 280, 227

van der Klis, M. 1989, in Timing Neutron Stars, ed. H. Ögelman \& E. P. J. van den Heuvel (Dordrecht: Kluwer), 27

Vaughan, B. A., et al. 1997, in preparation 